\title{
Intersection-Equivalence of Brownian Paths and Certain Branching Processes
}

\author{
Yuval Peres \\ Department of Statistics, University of California, Berkeley, CA 94120, USA \\ Email: peres@stat.berkeley.edu
}

Received: 11 January 1995

\begin{abstract}
We show that sample paths of Brownian motion (and other stable processes) intersect the same sets as certain random Cantor sets constructed by a branching process. With this approach, the classical result that two independent Brownian paths in four dimensions do not intersect reduces to the dying out of a critical branching process, and estimates due to Lawler (1982) for the long-range intersection probability of several random walk paths, reduce to Kolmogorov's 1938 law for the lifetime of a critical branching process. Extensions to random walks with long jumps and applications to Hausdorff dimension are also derived.
\end{abstract}

\section{Introduction}

Random walk and percolation problems in regular trees (sometimes called "Bethe lattices") are well known to be easier than the corresponding problems in Euclidean space. In this paper we show that long-range intersection probabilities for random walks, Brownian motion paths and Wiener sausages in Euclidean space, can be estimated up to constant factors by survival probabilities of branching processes and percolation processes on trees. The following "dictionary" illustrates the reduction.

\section{Problem in Euclidean space}

- How many (independent) Brownian paths in $\mathbf{R}^{d}$ can intersect?

- What is the probability that several random walk paths, started at random in a cube of side-length $2^{k}$, will intersect?

\section{Corresponding problem on trees}

- Which branching processes can have an infinite line of descent?

- What is the probability that a branching process survives for at least $k$ generations? 

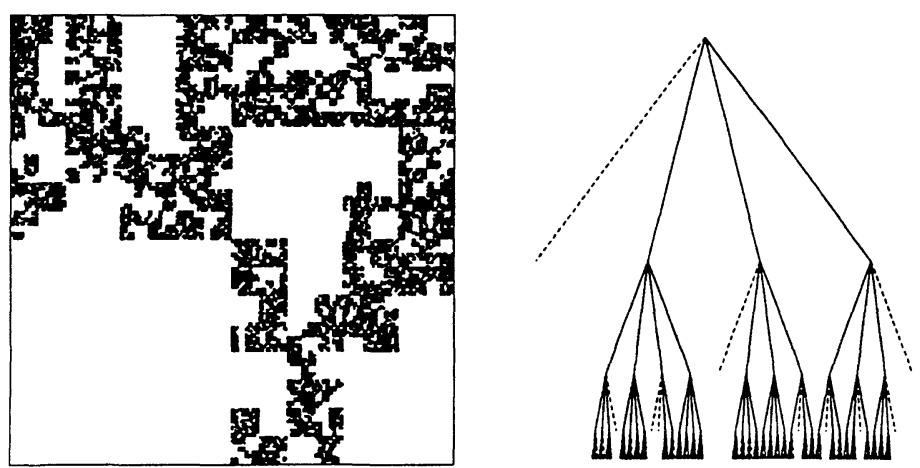

Fig. 1 Constructing $Q_{d}(p)$ for $d=2$ and $p=0.83$

- Which sets in $\mathbf{R}^{3}$ contain double points of Brownian motion?

- What is the Hausdorff dimension of the intersection of a fixed set in $\mathbf{R}^{d}$ with one or two Brownian paths?
- Which trees percolate at a fixed threshold $p$ ?

- What is the dimension of a percolation cluster on a general tree?

A set-valued random variable will be called a random set. (See the last section for measurability.) Interesting examples of random sets include the ranges of random walks and Brownian motion, percolation clusters, and the random fractals described below.

The following equivalence relation between random sets is crucial.

Definition 1. Two random (Borel) sets $A$ and $B$ in $\mathbf{R}^{d}$ are intersection-equivalent in the open set $U$, if for any closed set $\Lambda \subset U$, we have

$$
\mathbf{P}(A \cap \Lambda \neq \emptyset) \asymp \mathbf{P}(B \cap \Lambda \neq \emptyset) .
$$

In fact intersection-equivalence of $A$ and $B$ implies that (1) holds for all Borel sets $\Lambda$, by the Choquet Capacitability Theorem (see Carleson (1967), p. 3, or Dellacherie and Meyer (1978), III.28.)

The following well-known random recursive construction, sometimes called "fractal percolation," yields sets that are intersection-equivalent to sample paths. Given $d \geqq 3$ and $0<p<1$, consider the natural tiling of the unit cube $[0,1]^{d}$ by $2^{d}$ closed cubes of side $1 / 2$. Let $Z_{1}$ be a random subcollection of these cubes, where each cube has probability $p$ of belonging to $Z_{1}$, and these events are mutually independent. (Thus the cardinality $\left|Z_{1}\right|$ of $Z_{1}$ is a binomial random variable.) In general, if $Z_{k}$ is a collection of cubes of side $2^{-k}$, tile each cube $Q \in Z_{k}$ by $2^{d}$ closed subcubes of side $2^{-k-1}$ (with disjoint interiors) and include each of these subcubes in $Z_{k+1}$ with probability $p$ (independently). Finally, define

$$
Q_{d}(p)=\bigcap_{k=1}^{\infty} \bigcup_{Q \in Z_{k}} Q .
$$

${ }^{1}$ The symbol $\asymp$ means that the ratio of both sides is bounded above and below by positive constants which do not depend on $\Lambda$. 
In the construction of $Q_{d}(p)$, the cardinalities $\left|Z_{k}\right|$ of $Z_{k}$ form a Galton-Watson Branching process. Alternatively, the successive subdivisions into binary subcubes define a natural mapping from the regular tree of forward degree $2^{d}$, to the unit cube; the construction of $Q_{d}(p)$ corresponds to performing independent percolation with parameter $p$ on this tree and considering the set of infinite paths emanating from the root in its percolation cluster.

Theorem 1.1. Let $B_{d}(t)$ denote d-dimensional Brownian motion, started according to any fixed distribution with a bounded density for $B_{d}(0)$.

(i) If $d \geqq 3$ then the range $\left[B_{d}\right]=\left\{B_{d}(t): t \geqq 0\right\}$ is intersection-equivalent to $Q_{d}\left(2^{2-d}\right)$ in the unit cube.

(ii) More generally, let $S(t)$ be the symmetric stable process of index $\alpha$ in $\mathbf{R}^{d}$, started according to some fixed distribution with a bounded density. (See Kahane (1985) for background.) If $\alpha<d$ then the range $[S]$ is intersection-equivalent to $Q_{d}\left(2^{\alpha-d}\right)$ in the unit cube.

(iii) In dimension $d=2$, any Borel set $\Lambda$ such that $\mathbf{P}\left(Q_{2}(p) \cap \Lambda\right)>0$ for some $p<1$, satisfies $\mathbf{P}\left(\left[B_{2}\right] \cap \Lambda\right)=1$.

(Part (iii) is well known and is included for completeness).

Intersection-equivalence for random walks.

Definition 2. Let $\left\{S_{n}\right\}_{n \geqq 0}$ be a random walk on the lattice $\mathbf{Z}^{d}$, i.e., the differences $S_{n}-S_{n-1}$ are independent and identically distributed. We shall say that this random walk has Greenian index $\alpha$ if the Green function

$$
G(x, y):=\sum_{n=0}^{\infty} \mathbf{P}\left[S_{n}=y \mid S_{0}=x\right]
$$

satisfies $G(x, y) \asymp|x-y|^{\alpha-d}$ for all distinct $x, y \in \mathbf{Z}^{d}$. (That is, the ratio of the two sides is bounded between positive finite constants which may depend on the distribution of the random walk increments, but not on $x$ and $y$.)

It is well known that random walks with bounded increments of mean zero have Greenian index 2 , provided that $d \geqq 3$; for $d=3$, the boundedness assumption may be relaxed to finite variance (see Spitzer (1964)). Williamson (1968) shows that if $0<\alpha<\min \{2, d\}$, then any random walk $\left\{S_{n}\right\}$ in $\mathbf{Z}^{d}$ with increments satisfying $\mathbf{P}\left[S_{n}-S_{n-1}=x\right] \sim|x|^{-d-\alpha}$, has Greenian index $\alpha$.

Next, write $N=2^{k}$ and construct a random set of lattice points $Q_{d}(p ; k)$ by mimicking the construction of $Q_{d}(p)$. Partition the lattice points in $[0, N)^{d}$ to $2^{d}$ cubes of side $N / 2$. Each cube is independently retained with probability $p$. The retained cubes are subdivided similarly, and then each subcube is retained with probability $p$, etc. After $k$ stages we obtain individual lattice points, that constitute $Q_{d}(p ; k)$. This construction corresponds to percolation on a tree of depth $k$; the cardinality of $Q_{d}(p ; k)$ is the size of the $k^{\text {th }}$ generation in a branching process with offspring distribution Binomial $\left(2^{d}, p\right)$.

Theorem 1.2. Denote $N=2^{k}$. Let $\left\{S_{n}\right\}_{n \geqq 0}$ be a random walk on $\mathbf{Z}^{d}$ with Greenian index $\alpha<d$, where $S_{0}$ is uniformly distributed on the lattice points in $[0, N)^{d}$. 
Then for any $k$ and any set of lattice points $\Lambda \subset[0, N)^{d}$, we have

$$
c_{1} \leqq \frac{\mathbf{P}\left[\exists n \geqq 0: S_{n} \in \Lambda\right]}{\mathbf{P}\left[Q_{d}\left(2^{\alpha-d} ; k\right) \cap \Lambda \neq \emptyset\right]} \leqq c_{2},
$$

where $c_{1}, c_{2}$ are positive finite constants that do not depend on $k$ or $\Lambda$.

Theorem 1.3. Denote $N=2^{k}$. Let $\left\{S_{n}\right\}$ and $\left\{S_{m}^{\prime}\right\}$ be two independent random walks on the lattice $\mathbf{Z}^{d}$, started at random points uniformly distributed in $[0, N)^{d}$. Assume that $\left\{S_{n}\right\}$ and $\left\{S_{m}^{\prime}\right\}$ have Greenian indices $\alpha<d$ and $\beta<d$ respectively. Then

$$
\mathbf{P}\left[S_{n}=S_{m}^{\prime} \in[0, N)^{d} \text { for some } n, m \geqq 0\right] \asymp \begin{cases}1 & \text { if } \alpha+\beta>d \\ \frac{1}{\log N} & \text { if } \alpha+\beta=d \\ N^{\alpha+\beta-d} & \text { if } \alpha+\beta<d .\end{cases}
$$

In the special case of two simple random walks, $\alpha=\beta=2$ and Theorem 1.3 is included in more precise results of Lawler (1982), see Comment 5 in the last section. Felder and Fröhlich (1985) raised the question of estimating the intersection probability for random walks with long jumps, and solved a related model involving "random walk in dimension $(4-\varepsilon)$."

The rest of the paper is organized as follows. The next section contains applications of intersection-equivalence to Brownian paths and random walks. These include the proof of Theorem 1.3 and an extension to multiple intersections.

The proof of intersection-equivalence relies on three ingredients:

- The classical potential theory for Brownian motion (recalled in Sect. 3);

- The equivalence of capacity and percolation on trees established by R. Lyons (1992);

- The equivalence between capacities on a tree and in Euclidean space established in Benjamini and Peres (1992) and in Pemantle and Peres (1993).

The last two ingredients are described in Sect. 4, where Theorems 1.1 and 1.2 are proved. Section 5 contains applications to Hausdorff dimension of sample-path intersections and projections of random Cantor sets.

\section{Applications to Sample Path Intersections}

We shall need some basic facts about Galton-Watson branching processes (see, for instance, Athreya and Ney (1972)). Generation $k$ of the process has size $\left|Z_{k}\right|$, where $\left|Z_{0}\right| \equiv 1$ and $\left|Z_{1}\right|$ takes non-negative integer values; in general, $\left|Z_{k+1}\right|$ is a sum of $\left|Z_{k}\right|$ independent random variables with the same distribution as $\left|Z_{1}\right|$. We always assume that $\mathbf{P}\left[\left|Z_{1}\right|=1\right]<1$. If $\mathbf{E}\left|Z_{1}\right|>1$ then the process is supercritical and $\mathbf{P}\left[\left|Z_{k}\right|>0\right.$ for all $\left.k\right]>0$. If $\mathbf{E}\left|Z_{1}\right| \leqq 1$, then the process dies out, i.e., $\mathbf{P}\left[\left|Z_{k}\right|>\right.$ $0] \rightarrow 0$ as $k \rightarrow \infty$. Next, we recall a quantitative version of this.

Lemma 2.1 [Kolmogorov (1938)]. Let $\left\{\left|Z_{k}\right|\right\}$ be a branching process with $\left|Z_{1}\right|$ bounded.

(i) Critical case: If $\mathbf{E}\left|Z_{1}\right|=1$, then $\mathbf{P}\left[\left|Z_{k}\right|>0\right] \asymp 1 / k$.

(ii) Subcritical case: If $\mathbf{E}\left|Z_{1}\right|<1$ then $\mathbf{P}\left[\left|Z_{k}\right|>0\right] \asymp\left(E\left|Z_{1}\right|\right)^{k}$.

In fact, Kolmogorov proved a more precise estimate under moment assumptions on $\left|Z_{1}\right|$. The moment assumptions were relaxed by later authors-see Athreya and 
Ney (1972), pp. 19 and 45. The classical proof of Lemma 2.1 is a calculation with generating functions; a shorter proof for the case we need, in which $\left|Z_{1}\right|$ has a binomial distribution, is given in Sect. 4.

The usefulness of Theorem 1.1 is due to the next two easy lemmas.

Lemma 2.2. Suppose that $A_{1}, \ldots, A_{k}, F_{1}, \ldots, F_{k}$ are independent random Borel sets, with $A_{i}$ intersection-equivalent to $F_{i}$ for $1 \leqq i \leqq k$. Then $A_{1} \cap A_{2} \cap \ldots \cap A_{k}$ is intersection-equivalent to $F_{1} \cap F_{2} \cap \ldots \cap F_{k}$.

Proof. By induction, reduce to the case $k=2$. It clearly suffices to show that $A_{1} \cap A_{2}$ is intersection-equivalent to $F_{1} \cap A_{2}$, and this is done by conditioning on $A_{2}$ :

$$
\begin{aligned}
\mathbf{P}\left(A_{1} \cap A_{2} \cap \Lambda \neq \emptyset\right) & =\mathbf{E}\left[\mathbf{P}\left(A_{1} \cap A_{2} \cap \Lambda \neq \emptyset \mid A_{2}\right)\right] \asymp \\
\mathbf{E}\left[\mathbf{P}\left(F_{1} \cap A_{2} \cap \Lambda \neq \emptyset \mid A_{2}\right)\right] & =P\left(F_{1} \cap A_{2} \cap \Lambda \neq \emptyset\right) .
\end{aligned}
$$

Lemma 2.3. For any $0<p, q<1$, if $Q_{d}(p)$ and $Q_{d}^{\prime}(q)$ are statistically independent, then their intersection $Q_{d}(p) \cap Q_{d}^{\prime}(q)$ has the same distribution as $Q_{d}(p q)$.

Proof. This is immediate from the construction of $\left.Q_{d}(p)\right)$.

Now we can start reaping the corollaries.

\section{Corollary 2.5 [Dvoretzky, Erdös, Kakutani, Taylor].}

(i) For all $d \geqq 4$, two independent Brownian paths in $\mathbf{R}^{d}$ are disjoint almost surely (a.s.). (Except, of course, at their starting point if it is identical).

(ii) In $\mathbf{R}^{3}$, two independent Brownian paths intersect a.s., but three paths a.s. have no points of mutual intersection.

(iii) In $\mathbf{R}^{2}$, any finite number of independent Brownian paths have nonempty mutual intersection almost surely.

Remark. The proof of the corollary was completed in Dvoretzky, Erdös, Kakutani and Taylor (1957), following earlier work of Dvoretzky, Erdös and Kakutani (1950). A proof using the renormalization group method was given by Aizenman (1985). Proof.

(i) It suffices to consider $d=4$ and show that two independent Brownian paths $\left[B_{4}\right]$ and $\left[B_{4}^{\prime}\right]$ a.s. have no points of intersection in the unit cube, since countably many cubes cover $\mathbf{R}^{4}$. For any $\varepsilon>0$, the distribution of $B_{4}(\varepsilon)$ has a bounded density, so by Theorem 1.1 and Lemma 2.2,

$$
\begin{aligned}
& \mathbf{P}\left(\left\{B_{4}(t): t \geqq \varepsilon\right\} \cap\left\{B_{4}^{\prime}(s): s \geqq \varepsilon\right\} \cap[0,1]^{4} \neq \emptyset\right) \asymp \\
& \mathbf{P}\left[Q_{4}(1 / 4) \cap Q_{4}^{\prime}(1 / 4) \neq \emptyset\right]=\mathbf{P}\left[Q_{4}(1 / 16) \neq \emptyset\right]
\end{aligned}
$$

using Lemma 2.3 in the last equality. But $Q_{4}(1 / 16)$ is a.s. empty because critical branching processes die out. Thus $\left\{B_{4}(t): t \geqq \varepsilon\right\}$ and $\left\{B_{4}^{\prime}(s): s \geqq \varepsilon\right\}$ are a.s. disjoint, and since $\varepsilon>0$ is arbitrary, we are done.

(ii) Since $\left[B_{3}\right]$ is intersection-equivalent to $Q_{3}(1 / 2)$ in the unit cube, the intersection of three independent Brownian paths is intersection-equivalent in the cube to the random set $Q_{3}(1 / 2) \cap Q_{3}^{\prime}(1 / 2) \cap\left(Q_{3}^{\prime \prime}(1 / 2)\right.$, which has the same distribution as $Q_{3}(1 / 8)$. Again, a critical branching process is obtained, and hence the triple intersection is a.s. empty. On the other hand, $\left\{B_{3}(t): t \geqq t_{0}\right\} \cap\left\{B_{3}^{\prime}(s): s \geqq t_{0}\right\}$ is 
intersection-equivalent to $Q_{3}(1 / 4)$ in the unit cube, for any positive $t_{0}$. Since $Q_{3}(1 / 4)$ is defined by a supercritical branching process,

$$
\mathbf{P}\left[\left\{B_{3}(t): t \geqq t_{0}\right\} \cap\left\{B_{3}^{\prime}(s): s \geqq t_{0}\right\} \neq \emptyset\right]>0 .
$$

The scaling property of Brownian motion implies that this probability does not depend on $t_{0}$; therefore, by the Hewitt-Savage zero-one law it is 1 , and thus $\left[B_{3}\right] \cap$ $\left[B_{3}^{\prime}\right] \neq \emptyset$ a.s.,

(iii) This is quite similar to the above; to avoid repetition, we delay the proof to Corollary 5.2, where a stronger assertion is established.

Intersections of random walks: Proof of Theorem 1.3. By Theorem 1.2, the paths $\left\{S_{n}\right\}$ and $\left\{S_{m}^{\prime}\right\}$ are intersection-equivalent to $Q_{d}\left(2^{\alpha-d} ; k\right)$ and $Q_{d}^{\prime}\left(2^{\beta-d} ; k\right)$, respectively, in the cube $[0, N)^{d}$. Therefore, using Lemma 2.2,

$$
\begin{aligned}
\mathbf{P}\left(\exists n, m \geqq 0: S_{n}\right. & \left.=S_{m}^{\prime} \in[0, n)^{d}\right) \asymp \mathbf{P}\left[Q_{d}\left(2^{\alpha-d} ; k\right) \cap Q_{d}^{\prime}\left(2^{\beta-d} ; k\right) \neq \emptyset\right] \\
& =\mathbf{P}\left[Q_{d}\left(2^{\alpha+\beta-2 d} ; k\right) \neq \emptyset\right] .
\end{aligned}
$$

The cardinality of $Q_{d}(p ; k)$ is the size of the $k^{\text {th }}$ generation in a branching process of mean $2^{d} p$, so that

$$
\mathbf{P}\left[Q_{d}(p ; k) \neq \emptyset\right] \asymp \begin{cases}1 & \text { if } 2^{d} p>1 \\ \frac{1}{k} & \text { if } 2^{d} p=1 \\ \left(2^{d} p\right)^{k} & \text { if } 2^{d} p<1\end{cases}
$$

by Lemma 2.1. Setting $p=2^{\alpha+\beta-2 d}$ and recalling that $N=2^{k}$ concludes the proof.

Remark. In dimension $d \geqq 3$, the same argument applies to $r$ independent random walks $\left\{S_{n}^{(1)}\right\}, \ldots,\left\{S_{n}^{(r)}\right\}$, started at uniform random points in $[0, N)^{d}$, where $N=2^{k}$ and the $i^{\text {th }}$ walk has Greenian index $\alpha_{i}$. We obtain that

$\mathbf{P}$ (There exist $n_{1}, n_{2}, \ldots, n_{r}$ such that $S_{n_{1}}^{(1)}=S_{n_{2}}^{(2)}=\ldots=S_{n_{r}}^{(r)} \in[0, N)^{d}$ )

$$
\asymp \begin{cases}1 & \text { if } \alpha_{\mathrm{tot}}>d(r-1) \\ \frac{1}{k} & \text { if } \alpha_{\mathrm{tot}}=d(r-1) \\ N^{\alpha_{\mathrm{tot}}-(r-1) d} & \text { if } \alpha_{\mathrm{tot}}<d(r-1),\end{cases}
$$

where $\alpha_{\mathrm{tot}}=\sum_{i=1}^{r} \alpha_{i}$.

A continuous version of Theorem 1.3 was proved by Aizenman (1985) for near-intersections of Brownian paths. The following theorem extends it to stable processes. For any set $A \subset \mathbf{R}^{d}$, denote by $A(\varepsilon)$ its $\varepsilon$-thickening in the Euclidean norm: $A\langle\varepsilon\rangle=\left\{\mathbf{x} \in \mathbf{R}^{d}:\|x-y\|<\varepsilon\right.$ for some $\left.y \in A\right\}$. (It is easily seen that the choice of norm is immaterial for the next theorem.) When $A$ is a Brownian path, $A\langle\varepsilon\rangle$ is called a Wiener Sausage.

Theorem 2.6. Let $S^{(1)}, S^{(2)}, \ldots, S^{(r)}$ be $r$ independent symmetric stable processes in $\mathbf{R}^{d}$, with corresponding indices $\alpha_{1}, \alpha_{2}, \ldots, \alpha_{r}$ all less than $d$, and initial distributions 
with bounded densities. Then the probability that the corresponding $\varepsilon$-thickenings intersect in the unit cube is estimated by:

$$
\mathbf{P}\left(\bigcap_{i=1}^{r}\left[S^{(i)}\right]\langle\varepsilon\rangle \cap[0,1]^{d} \neq \emptyset\right) \asymp \begin{cases}1 & \text { if } \alpha_{\mathrm{tot}}>d(r-1) \\ \frac{1}{|\log \varepsilon|} & \text { if } \alpha_{\mathrm{tot}}=d(r-1) \\ \varepsilon^{\mathrm{tot}-d(r-1)} & \text { if } \alpha_{\mathrm{tot}}<d(r-1),\end{cases}
$$

where $\alpha_{\mathrm{tot}}=\sum_{i=1}^{r} \alpha_{i}$ and $0<\varepsilon<1 / 2$ is arbitrary.

Remark. In the case of two paths, their $\varepsilon$-thickenings intersect if and only if the paths are within distance $2 \varepsilon$ of each other; thus when $r=2$ and $\alpha_{1}=\alpha_{2}=2$, the above estimate is essentially contained in Proposition 5.2 of Aizenman (1985). (There, instead of intersecting the paths with a cube, they are killed at an exponential time.) The assumption on the initial distributions in the preceding theorem can be changed, e.g. the same estimates hold when all $r$ processes are started at distinct points.

Proof of Theorem 2.6. The proof is similar to the proof of Theorem 1.3 on random walks, after two preparatory steps.

Step 1. If $A$ and $F$ are intersection-equivalent random subsets of $U \subset \mathbf{R}^{d}$; then their $\varepsilon$-thickenings $A\langle\varepsilon\rangle$ and $F\langle\varepsilon\rangle$ are intersection-equivalent in $U$, with the same constants. This is immediate from the definitions, since $A(\varepsilon)$ intersects $\Lambda$ iff $A$ intersects $\Lambda(\varepsilon)$.

Step 2. Recall the random collection of cubes $Z_{k}$ in the $k^{\text {th }}$ stage of the construction of $Q_{d}(p)$. Denote

$$
\hat{Z}_{k}(p)=\bigcup_{Q \in Z_{k}} Q
$$

Claim. If $k=k(\varepsilon)$ is chosen to satisfy $\varepsilon / 2 \leqq 2^{-k(\varepsilon)} d^{1 / 2}<\varepsilon$, then $\hat{Z}_{k(\varepsilon)}(p)$ is intersection-equivalent to the $\varepsilon$-thickening of $Q_{d}(p)$ in the unit cube, and the implied constants are uniform in $\varepsilon$, provided that $\mathbf{E}\left|Z_{1}\right|=2^{d} p>1$.

One direction of this equivalence is obvious, since each cube in $Z_{k(\varepsilon)}(p)$ has diameter $\leqq \varepsilon$ and it contains a point of $Q_{d}(p)$ in its interior with a fixed positive probability (given $Z_{k}$ ). For the converse, observe that the $\varepsilon$-thickening of $Q_{d}(p)$ is covered by a constant $c(d)$ number of translates of $\hat{Z}_{k(\varepsilon)}(p)$.

Step 3. By the preceding steps and Lemma 2.2, the set $\bigcap_{i=1}^{r}\left[S^{(i)}\right]\langle\varepsilon\rangle$ is intersectionequivalent to the $k^{\text {th }}$ stage $\hat{Z}_{k}$ in the construction of $Q_{d}(p)$; where $p=\Pi_{i=1}^{r} 2^{\alpha_{i}-d}=$ $2^{\alpha_{\text {tot }}-r d}$ and $k=k(\varepsilon)=\log _{2}\left(\frac{1}{\varepsilon}\right)+O(1)$. This corresponds to the $k^{\text {th }}$ generation in a branching process of mean $2^{d} p=2^{\alpha_{\text {tot }}+(1-r) d}$, so the assertion of the theorem follows from Lemma 2.1 .

\section{Potential Theory Background}

Definition 3. Let $\Lambda$ be a metric space with the metric $|x-y|$. Let $K: \Lambda \times \Lambda \rightarrow$ $[0, \infty)$ be a Borel function. The K-energy of a finite Borel measure $\mu$ on $\Lambda$ is

$$
\mathscr{E}_{K}(\mu)=\iint_{A} K(x, y) d \mu(x) d \mu(y) .
$$


The $K$-capacity of $\Lambda$ is

$$
\operatorname{Cap}_{K}(\Lambda)=\left[\inf _{\mu} \mathscr{E}_{K}(\mu)\right]^{-1},
$$

where the infimum is over probability measures $\mu$ on $\Lambda$ and by convention, $\infty^{-1}=0$.

If $K(x, y)=|x-y|^{-\beta}$ then we write $\mathscr{E}_{\beta}$ for $\mathscr{E}_{K}$ and $\mathrm{Cap}_{\beta}$ for $\mathrm{Cap}_{K}$.

The following classical theorem relates capacity to dimension.

Theorem 3.1 (Frostman (1935)). For any Borel set $\Lambda$ in Euclidean space, the critical parameter inf $\left\{\beta>0 \mid \operatorname{Cap}_{\beta}(\Lambda)=0\right\}$ is exactly the Hausdorff dimension of $\Lambda$.

In fact Frostman proved this only for closed $\Lambda$. For a proof, see Carleson (1967) or Kahane (1985). At the critical parameter, the capacity of $\Lambda$ can be either positive or zero, but it vanishes if the Hausdorff measure in that dimension is $\sigma$-finite on $\Lambda$.

Kakutani (1944) discovered the connection of Brownian motion with capacity, and proved a qualitative version of the next proposition. The quantitative version given is a consequence of the probabilistic potential theory developed later by Hunt and Doob.

Proposition 3.2. Let $\left\{S_{t}\right\}_{t \geqq 0}$ be a transient Markov chain on a countable state space, or a transient symmetric stable process of index $\alpha<d$ in $\mathbf{R}^{d}$, with the initial distribution $\pi$. If there are positive numbers $M_{1}$ and $M_{2}$ such that the Green kernel $G(x, y)$ for the process satisfies $M_{1} \leqq \int G(x, y) d \pi(x) \leqq M_{2}$ for all $y \in \Lambda$, then

$$
M_{1} \operatorname{Cap}_{G}(\Lambda) \leqq \mathbf{P}_{\pi}\left[\exists t \geqq 0: S_{t} \in \Lambda\right] \leqq M_{2} \operatorname{Cap}_{G}(\Lambda)
$$

More specifically:

(i) If $\left\{S_{t}\right\}$ is a symmetric stable process of index $\alpha<d$ in $\mathbf{R}^{d}$, and the initial distribution $\pi$ has a bounded density on the unit cube, then

$$
\mathbf{P}_{\pi}\left[\exists t \geqq 0: S_{t} \in \Lambda\right] \asymp \operatorname{Cap}_{d-\alpha}(\Lambda),
$$

for any Borel set $\Lambda \subset[0,1]^{d}$.

(ii) If $\left\{S_{t}\right\}$ is a random walk on $\mathbf{Z}^{d}$ of Greenian index $\alpha<d$, and for some fixed $C$, the initial distribution $\pi$ on the discrete cube $\{0,1, \ldots, N-1\}^{d}$ assigns each point there at most mass $C / N^{d}$, then

$$
\mathbf{P}_{\pi}\left[\exists t \geqq 0: S_{t} \in \Lambda\right] \asymp N^{\alpha-d} \operatorname{Cap}_{d-\alpha}(\Lambda),
$$

where the implied constants do not depend on $N$ or on $\Lambda \subset\{0,1, \ldots, N-1\}^{d}$.

Proof. There exists a finite measure $v$ on $\Lambda$ (the "equilibrium measure") such that for any initial point $x$,

$$
\mathbf{P}_{x}\left[\exists t \geqq 0: S_{t} \in \Lambda\right]=\int_{\Lambda} G(x, y) d v(y)
$$

and

$$
v(\Lambda)=\operatorname{Cap}_{G}(\Lambda)
$$


In the discrete case, $v(y)$ can be identified as an escape probability:

$$
v(y)=\mathbf{P}_{y}\left\{S_{t} \notin \Lambda \text { for all } t \geqq 1\right\},
$$

and then (5) follows by a last-exit decomposition; for a probabilistic interpretation in the continuous case see Mckean (1965), Port and Stone (1971) or Chung (1973). Averaging (5) yields

$$
\mathbf{P}_{\pi}\left[\exists t \geqq 0: S_{t} \in \Lambda\right]=\iint_{\Lambda} G(x, y) d \pi(x) d v(y) .
$$

Now the hypothesis of the proposition implies that this is bounded above and below by $M_{2} v(\Lambda)$ and $M_{1} v(\Lambda)$, respectively; invoking (6) concludes the proof of the general assertion. Next, we pass to the specific cases:

(i) In this case $G(x, y)=|x-y|^{\alpha-d}$, so $G(x, y) \geqq d^{(\alpha-d) / 2}$ for all $x, y$ in $[0,1]^{d}$. Denote Lebesgue measure in $\mathbf{R}^{d}$ by $\mathscr{L}_{d}$ and let $C$ be a bound for the density of $\pi$. A straightforward integration yields

$$
\int G(x, y) d \pi(x) \leqq C \int_{[0,1]^{d}} G(x, y) d \mathscr{L}_{d}(x) \leqq C M_{2}
$$

for some $M_{2}$ that depends only on $d$ and $\alpha$, and for all $y \in[0,1]^{d}$. The assertion follows.

(ii) In this case $G(x, y) \asymp(1+|x-y|)^{\alpha-d}$, so clearly $G(x, y)$ is bounded below by a constant multiple of $N^{\alpha-d}$ for all $x, y$ in a cube of side $N$. To obtain an upper bound for the average of $G(\cdot, y)$, estimate the sum by an integral:

$$
\begin{aligned}
\int G(x, y) d \pi(x) & \leqq \frac{C}{N^{d}} \sum_{x}\{G(x, y): x \in\{0,1, \ldots, N-1\}\} \\
& \leqq \frac{C^{\prime}}{N^{d}} \int_{[0, N]^{d}}|v-y|^{\alpha-d} d v \leqq C^{\prime \prime} N^{\alpha-d}
\end{aligned}
$$

where $C^{\prime}$ and $C^{\prime \prime}$ do not depend on $N$. This completes the proof.

\section{Proof of Intersection-Equivalence}

The second ingredient in the proof of intersection-equivalence is a fundamental result of $\mathrm{R}$. Lyons concerning percolation on trees.

Notation. Let $T$ be a finite or infinite, rooted tree (i.e., a connected acyclic graph with a distinguished vertex $\rho$ designated as the root.) Maximal self-avoiding paths emanating from the root of $T$ are called rays, and the set of all rays is the boundary $\partial T$ of $T$. Rays can be infinite or finite; a finite ray necessarily terminates in a vertex of degree one, called a leaf of $T$. The number of edges that two rays $\xi$ and $\eta$ have in common is denoted by $|\xi \wedge \eta|$. The distance between two infinite rays $\xi$ and $\eta$ is defined to be $|\xi-\eta|:=2^{-|\xi \wedge \eta|}$. This motivates defining for any tree the $\beta$-energy of a measure $\mu$ on its boundary by

$$
\mathscr{E}_{\beta}(\mu):=\iint 2^{\beta|\xi \wedge \eta|} d \mu(\xi) d \mu(\eta)
$$




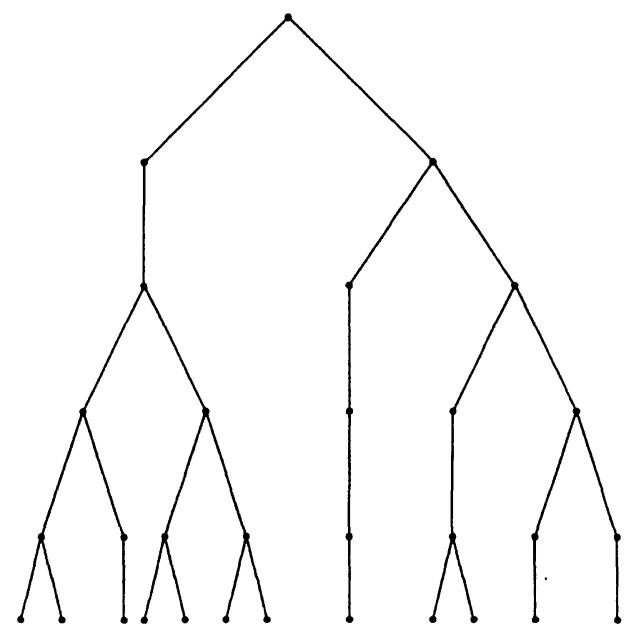

Fig. . 2 The first five levels of a tree

The constant 2 is convenient here because we use binary cubes in the application to Euclidean space.

Let $0<p<1$. Percolation with parameter $p$ on $T$ is obtained by removing each edge of $T$ with probability $1-p$ and, retaining it with probability $p$, with mutual independence among edges. Say that a ray $\xi$ survives the percolation if all the edges on $\xi$ are retained, and say that the tree boundary $\partial T$ survives if some ray of $T$ survives.

We can now state the result of Lyons that we need. An alternative proof, in which the percolation probability is interpreted as a hitting probability for a certain Markov chain, is given in Benjamini, Pemantle and Peres (1994).

Theorem 4.1 (Specialized from Theorem 2.1 of Lyons (1992)). Let $\beta>0$. If percolation at level $p=2^{-\beta}$ is performed on a rooted tree $T$, then

$$
\operatorname{Cap}_{\beta}(\partial T) \leqq \mathbf{P}[\partial T \text { survives the percolation }] \leqq 2 \operatorname{Cap}_{\beta}(\partial T) \text {. }
$$

Remarks. (i) The relation $p=2^{-\beta}$ arises from the definition of the energy in (8). (ii) For any vertex $\sigma$ of $T$, denote by $[\sigma]$ the set of rays going through $\sigma$, and by $|\sigma|$ the number of edges between $\sigma$ and the root. Using summation by parts, the energy of a probability measure $\mu$ on the boundary of a tree $T$ can be rewritten as

$$
\mathscr{E}_{\beta}(\mu)=1+\left(1-2^{-\beta}\right) \sum_{\sigma \in T} 2^{\beta|\sigma|} \mu[\sigma]^{2} .
$$

Example. A proof of Lemma 2.1 for a binomial offspring distribution. A branching process $\left\{Z_{j}\right\}$ with a binomial offspring distribution may be obtained from the regular $b$-ary tree $\Gamma$, by performing percolation at level $p$ and considering the connected component of the root. Denote by $\Gamma_{k}$ the $k^{\text {th }}$ level of $\Gamma$. Writing $p=2^{-\beta}$, we have

$$
\mathbf{P}\left(\left|Z_{k}\right|>0\right)=\mathbf{P}\left[\Gamma_{k} \text { survives the percolation }\right] \asymp \operatorname{Cap}_{\beta}\left(\Gamma_{k}\right) \text {. }
$$


For each $j$, the minimum of $\sum_{|\sigma|=j} \mu[\sigma]^{2}$ over probability measures $\mu$ on $\Gamma_{k}$ is $b^{-j}$, and is attained only by the uniform distribution. The expression (9) for the energy of measures implies that the uniform distribution on $\Gamma_{k}$ minimizes energy, and

$$
\operatorname{Cap}_{\beta}\left(\Gamma_{k}\right) \asymp\left(\sum_{j=0}^{k} 2^{\beta j} b^{-j}\right)^{-1}=\left(\sum_{j=0}^{k}(p b)^{-j}\right)^{-1} .
$$

Considering separately the critical case $p b=1$ and the subcritical case $p b<1$ concludes the proof.

Remark. In the example above, the traditional method of generating functions yields more precise estimates; the power of Theorem 4.1 lies in its applicability to general trees.

The third ingredient in the proof of intersection-equivalence relates the capacity on the tree boundary to capacity in Euclidean space. We employ the canonical mapping $\mathscr{R}$ from the boundary of a $2^{d}$-ary tree $\Gamma^{(2 d)}$ (every vertex has $2^{d}$ children) to the cube $[0,1]^{d}$. Formally, label the edges from each vertex to its children in a one-to-one manner with the vectors in $\Omega=\{0,1\}^{d}$. Then the boundary $\partial \Gamma^{(2 d)}$ is identified with the sequence space $\Omega^{\mathbf{Z}^{+}}$and we define $\mathscr{R}: \Omega^{\mathbf{Z}^{+}} \rightarrow[0,1]^{d}$ by

$$
\mathscr{R}\left(\omega_{1}, \omega_{2}, \ldots\right)=\sum_{n=1}^{\infty} 2^{-n} \omega_{n} .
$$

Similarly, a vertex $\sigma$ of $\Gamma^{\left(2^{d}\right)}$ is identified with a finite sequence $\left(\omega_{1}, \ldots, \omega_{k}\right) \in \Omega^{k}$ if there are exactly $k$ edges between the root and $\sigma$, and we write $\mathscr{R}(\sigma)$ for the cube of side $2^{-k}$ obtained as the image under $\mathscr{R}$ of all sequences in $\Omega^{\mathbf{Z}^{+}}$with prefix $\left(\omega_{1}, \ldots, \omega_{k}\right)$.

Theorem 4.2 (Benjamini and Peres (1992), Pemantle and Peres (1994)). With the notation above, let $T$ be a subtree of the regular $2^{d}$-ary tree $\Gamma^{\left(2^{d}\right)}$, so we may identify $\partial T$ with a subset of $\Omega^{\mathbf{Z}^{+}}$. Then for any finite measure $\mu$ on $\partial T$, and any $\beta>0$, we have

$$
\mathscr{E}_{\beta}(\mu) \asymp \mathscr{E}_{\beta}\left(\mu \mathscr{R}^{-1}\right)
$$

where the implied constants depend only on the dimension $d$. It follows that

$$
\operatorname{Cap}_{\beta}(\partial T) \asymp \operatorname{Cap}_{\beta}(\mathscr{R}(\partial T)) .
$$

Sketch of Proof. By (9), the energy of a measure $\mu$ on $\partial T$ satisfies

$$
\mathscr{E}_{\beta}(\mu) \asymp \sum_{k=0}^{\infty} 2^{\beta k} \sum_{|\sigma|=k} \mu[\sigma]^{2}
$$

(using the notation in Remark (ii) above). In the inner sum, every vertex $\sigma$ (that corresponds via $\mathscr{R}$ to a binary cube) "interacts" only with itself. A similar expression may be written for the energy of the measure $\mu \mathscr{R}^{-1}$ in Euclidean space; here every binary cube interacts with all binary cubes of the same size that touch it, so that

$$
\mathscr{E}_{\beta}\left(\mu \mathscr{R}^{-1}\right) \asymp \sum_{k=0}^{\infty} 2^{\beta k} \sum_{|\sigma|=|\tau|=k}\{\mu[\sigma] \mu[\tau]: \mathscr{R}(\sigma) \text { and } \mathscr{R}(\tau) \text { touch }\} .
$$


The obvious inequality $\mu[\sigma] \mu[\tau] \leqq \frac{1}{2}\left(\mu[\sigma]^{2}+\mu[\tau]^{2}\right)$, together with the key observation

$$
\#\{\tau \in T:|\tau|=|\sigma| \text { and } \mathscr{R}(\tau) \text { touches } \mathscr{R}(\sigma)\} \leqq 3^{d} \text { for all } \sigma \in T
$$

imply that the two energies in (13) and (14) are comparable.

The capacity assertion of the theorem follows, since any measure $v$ on $\mathscr{R}(\partial T) \subseteq$ $[0,1]^{d}$ can be written as $\mu \mathscr{R}^{-1}$ for an appropriate measure $\mu$ on $\partial T$.

Remark. We shall need a variant of Theorem 4.2 for finite trees, that has a similar proof. Denote $\Omega=\{0,1\}^{d}$. As above, we identify the $k^{\text {th }}$ level of the $2^{d}$-ary tree $\Gamma^{\left(2^{d}\right)}$ with $\Omega^{k}$. Let $N=2^{k}$, and consider the natural mapping $\mathscr{R}_{k}$ from $\Omega^{k}$ to the discrete cube $\{0,1, \ldots, N-1\}^{d}$, defined by

$$
\mathscr{R}_{k}\left(\omega_{1}, \ldots, \omega_{k}\right)=\sum_{j=1}^{k} 2^{k-j} \omega_{j} .
$$

Then any subset $F$ of $\Omega^{k}$ can be viewed as the boundary of a finite subtree of $\Gamma^{\left(2^{d}\right)}$ and we have

$$
\operatorname{Cap}_{\beta}(F) \asymp N^{-\beta} \operatorname{Cap}_{\beta}\left(\mathscr{R}_{k}(F)\right)
$$

Corollary 4.3. Let $\beta>0$ and $d \geqq 1$. Then

(i) For any closed set $\Lambda$ in the cube $[0,1]^{d}$ we have

$$
\mathbf{P}\left(Q_{d}\left(2^{-\beta}\right) \cap \Lambda \neq \emptyset\right) \asymp \operatorname{Cap}_{\beta}(\Lambda) .
$$

(ii) If $N=2^{k}$ then any subset $A$ of the discrete cube $\{0,1, \ldots, N-1\}^{d}$ satisfies

$$
\mathbf{P}\left(Q_{d}\left(2^{-\beta} ; k\right) \cap A \neq \emptyset\right) \asymp N^{-\beta} \operatorname{Cap}_{\beta}(A) .
$$

Proof. (i) Any closed set $\Lambda$ in the cube $[0,1]^{d}$ can be written as the image $\mathscr{R}(\partial T)$ of the boundary of some subtree $T$ of the regular $2^{d}$-ary tree, where $\mathscr{R}$ is the representation map (11). We perform percolation at level $p=2^{-\beta}$ on this tree. Then by Theorem 4.1 and Theorem 4.2,

$$
\begin{aligned}
\mathbf{P}\left[Q_{d}(p) \text { intersects } \Lambda\right] & =\mathbf{P}[\partial T \text { survives the percolation }] \\
& \asymp \operatorname{Cap}_{\beta}(\partial T) \asymp \operatorname{Cap}_{\beta}(\Lambda)
\end{aligned}
$$

(ii) This is proved in the same way, using the remark preceding the corollary.

Proof of Theorem 1.1. Part (i) is the special case $\alpha=2$ of part (ii), so we prove the latter. Denote by $\pi$ the distribution of $S_{0}$. Then by Proposition 3.2(i) and the previous corollary,

$$
\mathbf{P}_{\pi}\left[\exists t \geqq 0: S_{t} \in \Lambda\right] \asymp \operatorname{Cap}_{d-\alpha}(\Lambda) \asymp \mathbf{P}\left[Q_{d}(p) \text { intersects } \Lambda\right] .
$$

Part (iii) is more standard: If $\Lambda$ intersects $Q_{d}(p)$ for some $p<1$ with positive probability, then $\Lambda$ must have positive Hausdorff dimension (see Lemma 5.1 below). Therefore $\Lambda$ has positive logarithmic capacity and the conclusion follows, see Kakutani (1944b) or Kahane (1985). 
Proof of Theorem 1.2. The proof of Theorem 1.1 applies, using Proposition 3.2(ii) and Corollary 4.3(ii).

One direction of the following corollary was proved by Evans (1987) and Tongring (1988); the full equivalence was proved in a more general setting by Fitzsimmons and Salisbury (1989).

Corollary 4.4. Let $B_{3}$ and $B_{3}^{\prime}$ be two independent Brownian motions in $\mathbf{R}^{3}$, started according to distributions with bounded density in the unit cube. Then for any closed set $\Lambda$ in $[0,1]^{3}$ we have

$$
\mathbf{P}\left(\left[B_{3}\right] \cap\left[B_{3}^{\prime}\right] \cap \Lambda \neq \emptyset\right) \asymp \operatorname{Cap}_{1}(\Lambda) .
$$

Proof. By Theorem 1.1 and Lemma 2.2, the random set $\left[B_{3}\right] \cap\left[B_{3}^{\prime}\right]$ is intersectionequivalent to $Q_{3}(1 / 4)$. The assertion then follows from Corollary 4.3 .

\section{Applications to Hausdorff Dimension}

Lemma 5.1 (Hawkes 1981, Lyons 1990). Let $p=2^{-\beta}<1$. For any set $\Lambda \subset$ $[0,1]^{d}$ we have

(i) If $\operatorname{dim}(\Lambda)<\beta$ then $\Lambda \cap Q_{d}(p)$ is almost surely empty.

(ii) If $\operatorname{dim}(\Lambda)>\beta$ then $\Lambda$ intersects $Q_{d}(p)$ with positive probability.

(iii) If $\operatorname{dim}(\Lambda)>\beta$ then $\left\|\operatorname{dim}\left(\Lambda \cap Q_{d}(p)\right)\right\|_{\infty}=\operatorname{dim}(\Lambda)-\beta$, where the norm is an essential supremum in the underlying probability space.

Proof. Parts (i) and (ii) follow immediately from Corollary 4.3 and Frostman's Theorem connecting dimension and capacity (Theorem 3.1). Part (iii) is a consequence of the preceding parts and the fact that the intersection $Q_{d}(p) \cap Q_{d}^{\prime}(q)$ has the same distribution as $Q_{d}(p q)$.

In conjunction with Theorem 1.1, the lemma above yields short proofs of dimension formulae first established by J. Hawkes using very different methods.

Corollary 5.2 (Hawkes 1971a, 1971b). Let $\Lambda$ be a Borel set in $\mathbf{R}^{d}$, and let $\left[B_{d}\right]$ denote a Brownian path in $\mathbf{R}^{d}$. Then

(i) In dimension $d \geqq 3$ : If $\operatorname{dim}(\Lambda) \geqq d-2$ then $\left\|\operatorname{dim}\left(\Lambda \cap\left[B_{d}\right]\right)\right\|_{\infty}=\operatorname{dim}(\Lambda)$ $+2-d$.

(ii) Let $\left[B_{2}^{(1)}\right],\left[B_{2}^{(2)}\right], \ldots$ be independent Brownian paths in the plane. Then $\operatorname{dim}\left(\Lambda \cap\left[B_{2}^{(1)}\right] \cap \ldots \cap\left[B_{2}^{(k)}\right]\right)=\operatorname{dim} \Lambda$ a.s., for any finite $k \geqq 1$.

Remark. Part (ii) obviously strengthens Corollary 2.5(iii).

Proof. We may assume that $\Lambda$ is contained in $[0,1]^{d}$.

(i) Let $Q_{d}^{\prime}(p)$ be a copy of $Q_{d}(p)$ that is independent of $B_{d}$. Then Theorem 1.1 implies

$$
\begin{aligned}
\mathbf{P}\left(\Lambda \cap\left[B_{d}\right] \cap Q_{d}^{\prime}(p) \neq \emptyset\right) & \asymp \mathbf{P}\left(\Lambda \cap Q_{d}\left(2^{2-d}\right) \cap Q_{d}^{\prime}(p) \neq \emptyset\right) \\
& =\mathbf{P}\left(\Lambda \cap Q_{d}\left(2^{2-d} p\right) \neq \emptyset\right) .
\end{aligned}
$$

Now by Lemma 5.1 , the last probability is positive if $\operatorname{dim}(\Lambda)+\log _{2}\left(2^{2-d} p\right)>0$, and vanishes if $\operatorname{dim}(\Lambda)+\log _{2}\left(2^{2-d} p\right)<0$. Applying Lemma 5.1 to $\Lambda \cap B_{d}$ in place of $\Lambda$ completes part (i). 
(ii) By induction, it clearly suffices to prove the assertion for one planar Brownian path $\left[B_{2}\right]$. Given $q>2^{-\operatorname{dim}(\Lambda)}$, choose $p<1$ such that $p q>2^{-\operatorname{dim}(\Lambda)}$. Then

$$
\mathbf{P}\left(\Lambda \cap Q_{2}(q) \cap Q_{2}^{\prime}(p) \neq \emptyset\right)>0,
$$

and by Theorem 1.1, with positive probability $\Lambda \cap Q_{2}(q)$ intersects almost all Brownian paths. By Fubini's theorem,

$$
\mathbf{P}\left(\Lambda \cap\left[B_{2}\right] \cap Q_{2}(q) \neq \emptyset \mid\left[B_{2}\right]\right)>0 \text { a.s. , }
$$

so that by Lemma 5.1

$$
\operatorname{dim}\left(\Lambda \cap\left[B_{2}\right]\right)+\log _{2}(q)>0 \text { a.s. }
$$

Since this holds for any $q>2^{-\operatorname{dim}(\Lambda)}$, it follows that

$$
\operatorname{dim}\left(\Lambda \cap\left[B_{2}\right]\right) \geqq \operatorname{dim}(\Lambda) \text { a.s. }
$$

Remark. The same proof yields the following general result of Hawkes (1971b): Let $S^{(1)}, S^{(2)}, \ldots, S^{(k)}$ be independent symmetric stable processes in $\mathbf{R}^{d}$, where $S^{(i)}$ has index $\alpha_{i}<d$ for each $i \leqq k$. Then for any Borel set $\Lambda \subset \mathbf{R}^{d}$,

$$
\left\|\operatorname{dim}\left(\Lambda \cap\left[S^{(1)}\right] \cap \ldots \cap\left[S^{(k)}\right]\right)\right\|_{\infty}=\operatorname{dim}(\Lambda)-\sum_{i=1}^{k}\left(d-\alpha_{i}\right),
$$

provided the right-hand side is positive.

The dimension of $\left[S^{(1)}\right] \cap \ldots \cap\left[S^{(k)}\right]$ was computed earlier by Taylor (1966) and Fristedt (1967).

5.1. Projections of Random Cantor Sets. For certain random Cantor sets (more general than the sets $Q_{d}(p)$ ), Dekking and Grimmett (1988) found the Minkowski ( $\equiv$ box) dimension of their projections to the coordinate axes. The Hausdorff dimension of these projections was determined by Falconer (1988). The following corollary applies only to the sets $Q_{d}(p)$, but covers projections in any direction.

Corollary 5.3. Let $L$ be linear or affine map from $\mathbf{R}^{d}$ onto $\mathbf{R}^{k}$, and let $0<p<1$.

(i) If $d+\log _{2}(p)>k$, then the image $L\left(Q_{d}(p)\right)$ has positive $k$-dimensional Lebesgue measure a.s. on the event $Q_{d}(P) \neq \emptyset$.

(ii) If $0<d+\log _{2}(p) \leqq k$, then the image $L\left(Q_{d}(p)\right)$ has Hausdorff dimension $d+\log _{2}(p)$ a.s. on the event $Q_{d}(p) \neq \emptyset$.

\section{Remarks.}

- Denote by $\left|Z_{1}\right|$ the number of cubes in the first generation of the construction of $Q_{d}(p)$. We shall need the standard observation that the only non-negative fixed-points $x$ of the generating function

$$
f(x):=\sum_{j=0}^{2^{d}} \mathbf{P}\left(\left|Z_{1}\right|=j\right) x^{j}
$$

are 1 and the extinction probability of $Q_{d}(p)$. This fact is an immediate consequence of the convexity of $f$, and plays the role of a zero-one law. 
- Since $\operatorname{dim}\left[Q_{d}(p)\right]=d+\log _{2}(p)$ a.s. upon nonextinction, general results of Marstrand (1954) and Mattila (1975) imply that the assertions of the corollary hold for almost all orthogonal projections $L$ onto $k$-dimensional linear subspaces of $\mathbf{R}^{d}$ (with respect to the natural rotation-invariant measure on the Grassmann manifold of $k$-dimensional subspaces).

- The proof of the corollary is especially short when $L$ is an orthogonal projection and $d+\log _{2}(p) \leqq 2$, since in this case $Q_{d}(p)$ is intersection-equivalent to the range of a symmetric stable process in $\mathbf{R}^{d}$, and the projection of that range to $\mathbf{R}^{k}$ is the range of a symmetric stable process in $\mathbf{R}^{k}$.

Proof. By composing $L$ with a dilation and a translation if necessary, we may assume that $L$ maps $[0,1]^{d}$ into the unit cube of $\mathbf{R}^{k}$.

(i) For every point $y \in L\left((0,1)^{d}\right)$, the preimage $L^{-1}(y)$ has Hausdorff dimension $d-k$ and thus intersects $Q_{d}(p)$ with positive probability by Lemma 5.1. Fubini's Theorem implies that the expected $k$-dimensional volume of $L\left(Q_{d}(p)\right)$ is positive. By conditioning on $\left|Z_{1}\right|$, we see that $\mathbf{P}$ (the $k$-dimensional volume of $L\left(Q_{d}(p)\right)$ vanishes) is a fixed point of the generating function $f$ in (16), so this probability must equal the extinction probability of $Q_{d}(p)$.

(ii) We only need to prove the lower bound on dimension. Let $\gamma<d+\log _{2}(p)$, so that $Q_{k}\left(2^{-\gamma}\right)$ has dimension $k-\gamma$ a.s. upon nonextinction. Therefore the preimage $L^{-1}\left(Q_{k}\left(2^{-\gamma}\right)\right)$ has dimension greater than $\log _{2}(1 / p)$, and hence intersects $Q_{d}(p)$ with positive probability. In other words, $L\left(Q_{d}(p)\right)$ intersects $\left(Q_{k}\left(2^{-\gamma}\right)\right)$ with positive probability, so its dimension must be at least $\gamma$ with positive probability. Since $\mathbf{P}\left[\operatorname{dim} L\left(Q_{d}(p)\right) \leqq \gamma\right]$ is a fixed-point of the generating function in (16), this concludes the proof.

\section{Comments on the Literature}

1. For random closed sets in Euclidean space, the easiest way to introduce the needed measurability hypothesis is via the Hausdorff metric and the corresponding Borel $\sigma$-field. For a more general random set $A(\omega)$ in Euclidean space, one requires that the indicator of $A$ is jointly measurable as a function of $\omega$ and the space variable. Further measurability questions are then settled via the theory of Suslin sets and Choquet capacities. (See Dellacherie and Meyer (1978), VI.8 and VI.31.)

2. Aizenman (1985) suggested that intersections of Brownian paths, and percolation processes on trees, should be closely related. However, as pointed out in Aizenman's paper, attempting a direct probabilistic link between the two settings runs into delicate dependence problems. Here potential theory serves as a bridge.

3. The random fractals $Q_{d}(p)$ were described by Mandelbrot (1974), and natural measures on them were analyzed by Kahane and Peyriere (1976). The Hausdorff dimension of "branching sets" that include $Q_{d}(p)$ was determined by Hawkes (1981), and the exact Hausdorff measure in more general random constructions was found by Graf, Mauldin and Williams (1988). Chayes, Chayes and Durrett (1988) noted that the dimension of $Q_{d}(p)$ is easily inferred from the results of Kahane and Peyriere, and proved the remarkable fact that $Q_{d}(p)$ has large connected components if $p$ is sufficiently close to 1 . Further results in this direction were obtained by Dekking and Meester (1990)

4. In one dimension, the ranges of certain subordinators can be constructed by removing from the line random cutouts, that are determined by a Poisson point 
process in the plane. This was used by Mandelbrot (1972) and Hawkes (1977) to determine the intersection properties of these ranges.

5. The problem of estimating intersection probabilities for paths of simple random walk was raised by Erdős and Taylor (1960). The first solution was found by Lawler (1982), and alternative approaches were given later by Felder and Fröhlich (1985) and by Park (1989). The advantage of Lawler's method is that it yields much more precise asymptotics for the "long-range" intersection probabilities. These asymptotics are crucial in obtaining estimates of non-intersection probabilities for two independent simple random walks started at the same point ("short-range intersections"); see Lawler $(1985,1991)$ for a complete account. In our Theorem 1.3 , the intersection probabilities are only estimated up to constant factors, so the intersection-equivalence method does not seem useful for estimating non-intersection probabilities when they are small. Precise asymptotics for intersection and nonintersection probabilities of Wiener Sausages in $\mathbf{R}^{4}$ were recently obtained by Albeverio and Zhou (1993).

6. For concreteness, we have stated our continuous-time results for symmetric stable processes only, but they apply to any process $\left\{S_{t}\right\}$ that satisfies (3) for all closed sets $\Lambda$ in the cube - See Port and Stone (1971) and Hawkes (1979) for the potential theory of general Lèvy processes.

7. The idea of determining the dimension of a set from its intersection properties with other random sets was first used by Taylor (1966) to study multiple points of stable processes. The random sets used there were ranges of other stable processes. See Taylor (1986) for an insightful survey of the dimension theory of Lèvy processes.

8. A more precise version of Theorem 1.1 for planar Brownian motion is given in Peres (1994). The range of planar Brownian motion (started uniformly in $[0,1]^{2}$ and run for unit time) is intersection-equivalent in the unit square to a random set $Q_{2}^{*}$ that is constructed just like the sets $Q_{2}(p)$ but with scale-dependent retention probabilities: a binary square of side $2^{-k}$ is retained with probability $k /(k+1)$, given that its parent square of side $2^{1-k}$ was retained.

Acknowledgements. I am indebted to Chris Bishop for stating a conjecture that led to this work, and to Michael Aizenman, Itai Benjamini, Greg Lawler, Russell Lyons and Robin Pemantle for useful discussions. I thank Ofer Licht for Fig. 1.

\section{References}

1. Aizenman, M.: The intersection of Brownian paths as a case study of a renormalization method for quantum field theory. Commun. Math. Phys. 97, 91-110 (1985)

2. Albeverio, S., Zhou, X.Y.: Intersections of random walks and Wiener sausages in four dimensions. Preprint, 1993

3. Athreya, K., Ney, P.: Branching Processes. Berlin, Heidelberg, New York: Springer, 1972

4. Benjamini, I., Pemantle, R., Peres, Y.: Martin capacity for Markov chains. (To appear Ann. Probab.), 1994

5. Benjamini, I., Peres, Y.: Random walks on a tree and capacity in the interval. Ann. Inst. Henri Poincaré 28, 557-592 (1992)

6. Carleson, L.: Selected Problems on Exceptional Sets. Princeton, NJ: Van Nostrand, 1967

7. Chayes, J., Chayes, L., Durrett, R.: Connectivity properties of Mandelbrot's percolation process. Probab. Th. Rel. Fields 77, 307-324 (1988) 
8. Chung, K.L.: Probabilistic approach in potential theory to the equilibrium problem. Ann. Inst. Fourier, Grenoble 23, 313-322 (1973)

9. Dekking, M., Grimmett, G.R.: Superbranching processes and projections of random Cantor sets. Probab. Th. Rel. Fields 78, 335-355 (1988)

10. Dekking, M., Meester, R.: On the structure of Mandelbrot's percolation process and other random Cantor sets. J. Stat. Phys. 58, 1109-1126 (1990)

11. Dellacherie, C., Meyer, P.A.: Probabilities and Potential A. North-Holland Mathematics Studies 29, Paris: Hermann, 1978

12. Dvoretsky, A., Erdös, P., Kakutani, S.: Double points of paths of Brownian motion in n-space. Acta Sci Math. Szeged 12, 75-81 (1950)

13. Dvoretsky, A., Erdös, P., Kakutani, S., Taylor, S.J.: Triple points of Brownian motion in 3-space. Proc. Comb. Phil. Soc. 53, 856-862 (1957)

14. Evans, S.: Multiple points in the sample paths of a Lévy process. Probab. Th. Rel. Fields 76, 359-376 (1987)

15. Erdös, P., Taylor, S.J.: Some intersection properties of random walk paths. Acta. Math. Sci. Hung. 11, 231-248 (1960)

16. Falconer, K.J.: Projections of random Cantor sets. J. of Theoretical Probab. 2, 65-70 (1988)

17. Felder, G., Frölich, J.: Intersection properties of simple random walks: A renormalization group approach. Commun. Math. Phys. 97, 111-124 (1985)

18. Fitzsimmons, P.J., Salisbury, T.: Capacity and energy for multiparameter Markov processes. Ann. Inst. Henri Poincarè, Probab. 25, 325-350 (1989)

19. Fristedt, B.: An extension of a theorem of S.J. Taylor concerning the multiple points of the symmetric stable process. Zeits. Wahr. Verw. Geb. 9, 62-64 (1967)

20. Frostman, O.: Potential d'équilibre et capacité des ensembles. Thesis, Lund, 1935

21. Graf, S., Mauldin, R.D., Williams, S.C.: The exact Hausdorff dimension in random recursive constructions. Memoirs, the American Mathematical Society, Providence, Rhode Island, 1988

22. Hawkes, J.: On the Hausdorff dimension of the intersection of the range of a stable process with a Borel set. Zeits. Wahr. Verw. Geb. 19, 90-102 (1971a)

23. Hawkes, J.: Some dimension Theorems for the sample functions of stable processes. Indiana University Math. J. 20, 733-738 (1971b)

24. Hawkes, J.: Intersections of Markov random sets. Zeits. Wahr. Verw. Geb. 37, 243-251 (1977)

25. Hawkes, J.: Potential theory of Lévy processes. Proc. London Math. Soc. (3) 38, 335-352 (1979)

26. Hawkes, J.: Trees generated by a simple branching process. J. London Math. Soc. 24, 373-384 (1981)

27. Hunt, G.A.: Markov processes and potentials III. Illinois J. Math 2, 151-213 (1958)

28. Kahane, J.P.: Some random series of functions. Second edition, Cambridge: Cambridge University Press, 1985

29. Kahane, J.P., Peyriere, J.: Sur certaines martingales de B. Mandelbrot. Advances in Math. 22, 131-145 (1976)

30. Kakutani, S.: On Brownian motion in $n$-space. Proc. Imp. Acad. Tokyo 20, 648-652 (1944a)

31. Kakutani, S.: Two dimensional Brownian motion and harmonic functions. Proc. Imp. Acad. Tokyo 20, 706-714 (1944b)

32. Kolmogorov, A.N.: On the solution of a problem in biology, Izv. NII Matem. Mekh. Tomskogo Univ. 2, 7-12 (1938)

33. Lawler, G.: The probability of intersection of independent random walks in four dimensions. Commun. Math. Phys. 86, 539-554 (1982)

34. Lawler, G.: Intersections of random walks in four dimensions II. Commun. Math. Phys. 97, 583-594 (1985)

35. Lawler, G.: Intersections of random walks. Boston: Birkhäuser, 1991

36. Lyons, R.: Random walks and percolation on trees. Ann. Probab. 18, 931-958 (1990)

37. Lyons, R.: Random walks, capacity and percolation on trees. Ann. Probab. 20, 2043-2088 (1992)

38. Mandelbrot, B.B.: Renewal sets and random cutouts. Zeits. Wahr. verw. Geb. 22, 145-157 (1972)

39. Mandelbrot, B.B.: Intermittent turbulence in self-similar cascades: Divergence of high moments and dimension of the carrier. J. Fluid Mech. 62, 331-358 (1974)

40. Marstrand, J.M.: Some fundamental geometrical properties of plane sets of fractional dimensions. Proc. London Math. Soc. 4, 257-302 (1954) 
41. Mattila, P.: Hausdorff dimension, orthogonal projections and intersections with planes. Ann. Acad. Sci. Fenn., Ser. A, Math. 1, 227-244 (1975)

42. Mckean, H.P.: A probabilistic interpretation of equilibrium charge distribution. J. Math. Kyoto University 4, 617-623 (1965)

43. Park, Y.: Direct estimates on intersection probabilities of random walks. J. Stat. Phys. 57, 319-331 (1989)

44. Pemantle, R., Peres, Y.: Galton-Watson trees with the same mean have the same polar sets. (To appear in Ann. Probab, July 1995) (1994)

45. Peres, Y.: The percolation approach to capacity and intersections of random sets. In preparation, 1994

46. Port, S., Stone, C.: Infinitely divisible processes nad their potential theory I. Ann. Inst. Fourier (Grenoble) 21 (4), 179-265 (1971)

47. Taylor, S.J.: Multiple points for the sample paths of the symmetric stable process. Zeits. Wahr. Verw. Geb. 5, 247-264 (1966)

48. Taylor, S.J.: The measure theory of random fractals. Math. Proc. Camb. Phil. Soc. 100, 383406 (1986)

49. Tongring, N.: Which sets contain multiple points of Brownian motion? Math. Proc. Camb. Phil. Soc. 103, 181-187 (1988)

50. Williamson, J.A.: Random walks and Riesz kernels. Pacific J. Math. 25, 393-415 (1968)

Communicated by B. Simon 